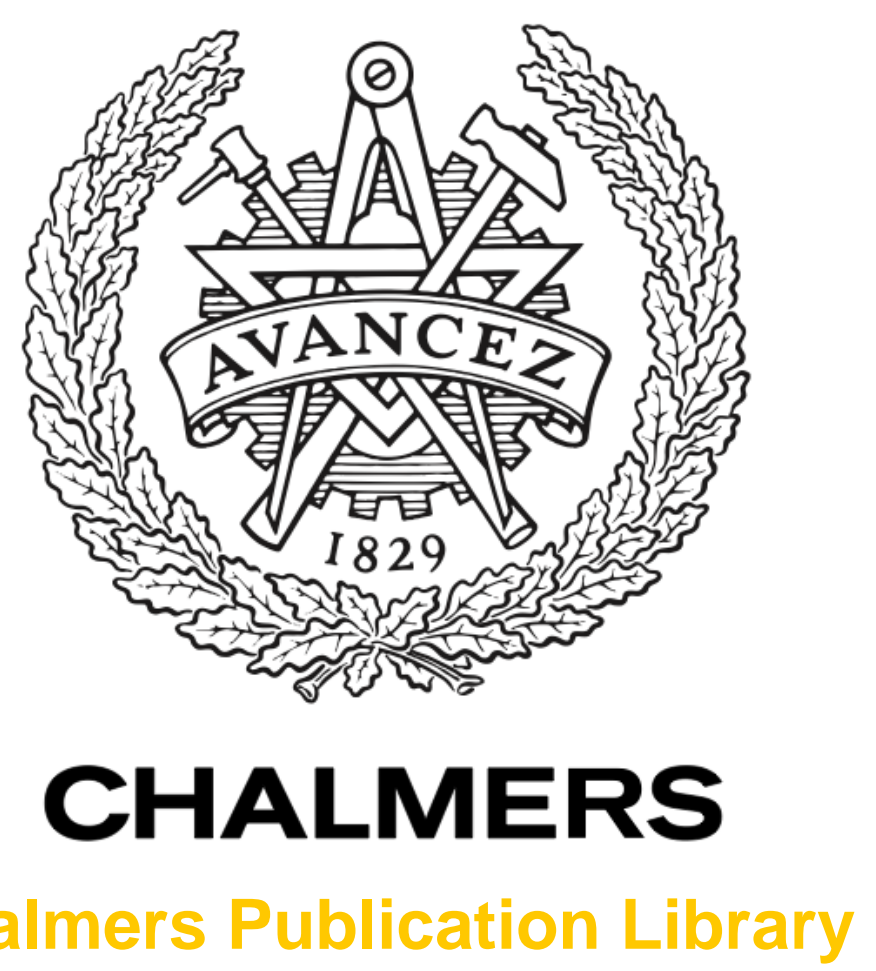

\title{
Spatially-Coupled Codes for Optical Communications: State-of-the-Art and Open Problems
}

This document has been downloaded from Chalmers Publication Library (CPL). It is the author's version of a work that was accepted for publication in:

20th OptoElectronics and Communications Conference, OECC 2015

Citation for the published paper:

Graell i Amat, A. ; Häger, C. ; Brännström, F. et al. (2015) "Spatially-Coupled Codes for Optical Communications: State-of-the-Art and Open Problems". 20th OptoElectronics and Communications Conference, OECC 2015

Downloaded from: http://publications.lib.chalmers.se/publication/217254

Notice: Changes introduced as a result of publishing processes such as copy-editing and formatting may not be reflected in this document. For a definitive version of this work, please refer to the published source. Please note that access to the published version might require a subscription. 


\title{
Spatially-Coupled Codes for Optical Communications: State-of-the-Art and Open Problems
}

\author{
(Invited Paper) \\ Alexandre Graell i Amat, Christian Häger, Fredrik Brännström, and Erik Agrell \\ Department of Signals and Systems, Chalmers University of Technology, SE-412 96 Gothenburg, Sweden
}

\begin{abstract}
We give a brief survey of a particularly interesting class of codes, called spatially-coupled codes, which are strong candidates for future optical communication systems. We discuss some recent research on this class of codes in the area of optical communications, and summarize some open research problems.
\end{abstract}

\section{INTRODUCTION}

The ever-growing demand in network capacity has made the use of forward error correction (FEC) a must in optical communications. The first FEC code proposals, in the late 1980s and early 1990s, were based on classical algebraic codes, mainly Reed-Solomon (RS) codes, and hard-decision decoding (HDD). RS codes are characterized by a large minimum distance, which allows to support error rates below $10^{-15}$, as required in optical communications. Furthermore, they possess a strong algebraic structure which can be exploited for lowcomplexity syndrome-based decoding. However, RS codes perform far away from the Shannon limit at low error rates.

The advent of turbo codes and the rediscovery of lowdensity parity-check (LDPC) codes, with unprecedented performance close to the Shannon limit, has attracted a significant interest in the optical communications community in the last few years in modern FEC schemes. Turbo and LDPC codes can be decoded with relatively low complexity using softdecision decoding (SDD), also referred to as belief propagation (BP) decoding. Today, coding for long-haul optical communications can be divided into two main active areas. On one hand, code constructions based on algebraic codes, such as turbo product codes, staircase codes [1] and tightly-braided block (TBB) codes [2], decoded using HDD. On the other hand, LDPC codes with SDD. The latter can provide an extra coding gain with respect to HDD schemes, at the expense of an increased decoding complexity and power consumption.

Recently, the spatial coupling of LDPC codes [3] has revealed itself as a powerful technique to construct codes that achieve capacity universally over the class of binary-input memoryless symmetric channels. The main principle behind this outstanding behavior is the remarkable fact that the $\mathrm{BP}$ threshold saturates to the maximum a posteriori (MAP) threshold of the underlying block code ensemble when the block length tends to infinity [3]. In plain words, the suboptimal BP decoder performs as well as the optimal MAP decoder.

A drawback of spatially-coupled LDPC (SC-LDPC) codes is the need for very large block lengths (100K bits and above) to yield the promised gains. This may be, in principle, a limiting factor for its use in applications such as wireless communications, characterized by relatively short packages. However, due to the very high data rates, optical communications can tolerate very large block lengths (a decoding delay of $10 \mu$ s translates to a processing delay of one million bits for a data rate of $100 \mathrm{~Gb} / \mathrm{s}$ ). Therefore, optical communications is one of the most appealing applications for this class of codes.

Spatial coupling is a very general concept. In the realm of coding, it can be applied to virtually any code with both HDD and SDD. For instance, staircase codes [1] and TBB codes [2] can be seen as particular cases of spatial coupling, in particular as an instance of spatially-coupled generalized LDPC (SC-GLDPC) codes $^{1}$.

In this paper, we review the class of spatially-coupled (SC) codes, discuss some recent developments on this class of codes in the optical communications arena, and point to some open research problems. Due to space constraints, our main focus is on SC-LDPC codes with SDD, but we also discuss some recent research on SC-GLDPC and HDD.

\section{LDPC AND SC-LDPC CODES}

A binary LDPC code encoding information messages of length $k_{\mathcal{C}}$ bits into codewords of length $n_{\mathcal{C}}$ bits is defined via an $r_{\mathcal{C}} \times n_{\mathcal{C}}$ sparse binary parity-check matrix $\mathbf{H}=\left[h_{i, j}\right]$, where $r_{\mathcal{C}} \geq n_{\mathcal{C}}-k_{\mathcal{C}}$ with equality if and only if $\mathbf{H}$ has full rank. One popular method to construct LDPC codes is by using protographs. Protograph-based codes are very attractive from a design perspective and allow for a highspeed hardware implementation, suitable for fiber-optical communications. A protograph is a bipartite graph defined by an $r_{\mathcal{C}}^{\prime} \times n_{\mathcal{C}}^{\prime}$ adjacency matrix $\mathbf{P}=\left[p_{i, j}\right]$, called the base matrix, with nonnegative entries. Given $\mathbf{P}$, the parity-check matrix $\mathbf{H}$ is obtained by replacing each entry $p_{i, j}$ with a random binary $M \times M$ matrix which contains $p_{i, j}$ ones in each row and column. This procedure is called lifting and $M \geq \max _{i, j} p_{i, j}$ is the so-called lifting factor. The design rate of the code is given by $R=1-r_{\mathcal{C}} / n_{\mathcal{C}}=1-r_{\mathcal{C}}^{\prime} / n_{\mathcal{C}}^{\prime}$, where $r_{\mathcal{C}}=r_{\mathcal{C}}^{\prime} M$ and $n_{\mathcal{C}}=n_{\mathcal{C}}^{\prime} M$. The code overhead (in \%) is given by $\mathrm{OH}=100 \cdot(1-R) / R$.

SC-LDPC codes are a class of LDPC codes with an embedded convolutional structure, i.e., they introduce memory in the encoding. SC-LDPC codes can also be constructed using protographs. The base matrix of a $(J, K)$ regular SC-LDPC

\footnotetext{
${ }^{1}$ GLDPC codes are a superclass of LDPC codes where the code constraints are linear block codes. LDPC codes are a particular class of GLDPC codes where the code constraints are simple parity-check codes.
} 


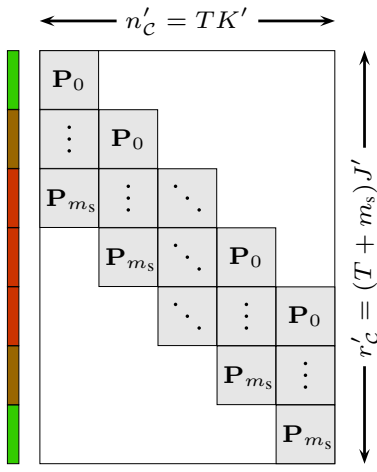

(a) terminated

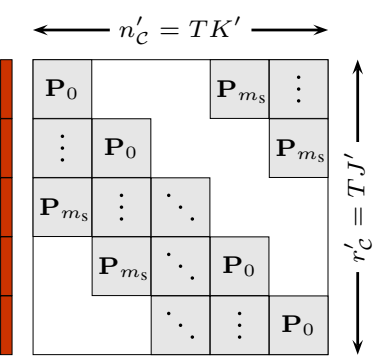

(b) tailbiting
Fig. 1. Base matrices $\mathbf{P}$ for protograph-based SC-LDPC codes.

is constructed by specifying matrices $\mathbf{P}_{i}, 0 \leq i \leq m_{\mathrm{s}}$, of dimension $J^{\prime} \times K^{\prime}$, where $m_{\mathrm{s}}$ is referred to as the memory. SC-LDPC codes are characterized by a semi-infinite paritycheck matrix with a band-diagonal structure. Therefore, as convolutional codes, they can generate infinitely long codewords. In practice, however, SC-LDPC codes need to be terminated to operate with a finite block length. Given the matrices $\mathbf{P}_{i}$, and the spatial length $T$, one can construct $\mathbf{P}$ as shown in Fig. 1(a) and Fig. 1(b) for two different ways of terminating the code. The resulting codes are called terminated and tailbiting, respectively. The matrices $\mathbf{P}_{i}$ are such that $\sum_{i=0}^{m_{\mathrm{s}}} \mathbf{P}_{i}$ has column weight $J$ and row weight $K$ for all columns and rows.

Spatial coupling can be easily visualized using protographs. Begin with the protograph of an LDPC code. The protograph of an SC-LDPC code is then constructed by copying this protograph a number of times and properly connecting the copies.

Example 1: Consider the $(3,6)$ regular SC-LDPC code with $\mathbf{P}_{0}=\mathbf{P}_{1}=\mathbf{P}_{2}=(1,1), T=5, J^{\prime}=1, K^{\prime}=2$, and $m_{\mathrm{s}}=2$. The protographs corresponding to the terminated and tailbiting cases are shown in Fig. 2(b) and Fig. 2(c). The protographs are constructed by copying 5 times the protograph of the $(3,6)$ regular LDPC code (shown in Fig. 2(a)) and connecting each protograph to the one to the left, and to the one to the right. The overhanging edges at the boundaries are then connected to extra parity-check nodes (terminated) or to the check nodes at the other extreme of the chain (tailbiting case).

Terminated and tailbiting SC-LDPC codes have design rates $R(T)=1-J^{\prime} / K^{\prime}-m_{\mathrm{s}} J^{\prime} /\left(T K^{\prime}\right)$ and $R=1-J^{\prime} / K^{\prime}$, respectively (for the codes in Example 1, $R(5)=0.3$ and $R=$ $0.5)$. Note that the terminated code has a lower rate. This rate loss arises from the fact that the check nodes at the boundaries of the coupling chain have lower degree, i.e., are connected to fewer variable nodes (see Fig. 2(b)). At the same time, the lower degree of check nodes at the boundaries leads to a locally better decoding capability (visualized with the green color in Fig. 1(a)), which is at the basis of the outstanding performance of SC-LDPC codes: This termination boundary effect initiates a wave-like decoding behavior from the exterior of the coupled chain to the interior. Note that the rate loss

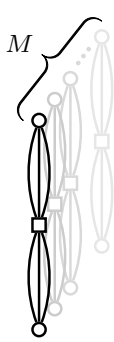

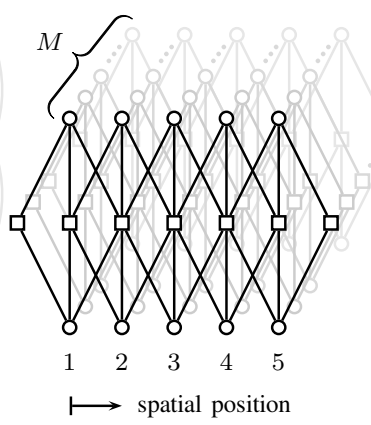

(b) terminated

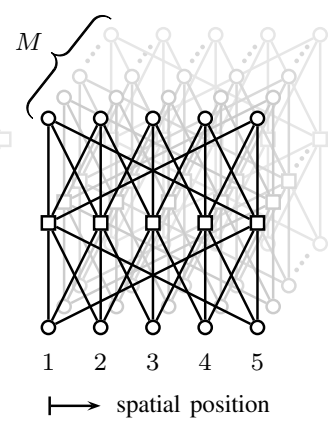

(c) tailbiting (a) regular

Fig. 2. Protographs for the regular LDPC code and SC-LDPC code with $T=5$ in Ex. 1. The first step of the lifting procedure to obtain the Tanner graph (i.e., copying the protograph $M$ times) is indicated in light gray.

can be made arbitrary small by letting $T \rightarrow \infty$, but this also leads to very long block lengths $n_{\mathcal{C}}=T K^{\prime} M$ (assuming a fixed lifting factor $M$ ). On the other hand, tailbiting does not entail a rate loss. However, by default, tailbiting codes do not experience a boundary effect and perform identically as the underlying LDPC block codes.

SC-LDPC can be decoded using standard SDD on the entire graph. However, due to the long code blocks, doing so would entail a large decoding delay and high complexity, which are not acceptable in optical communications. In order to alleviate this, a window decoder (WD) can be used. The WD restricts message updates in the decoding process to a window of the entire graph. After a predetermined number of decoding iterations, the decoding window slides to the next position. The WD significantly reduces the decoding delay with respect to standard SDD on the entire graph.

\section{Some Results ON SC CODES}

Significant research has been conducted in the recent years on SC codes with SDD for optical communications, see [4-7] and references therein. In [6] SC-LDPC codes with matrices $\mathbf{P}_{i}$ (see Fig. 1) with irregular degree distributions were proposed. The idea is to optimize the degree distribution in order to improve the performance for a given number of iterations within the decoding window (i.e., for a given decoding delay). To achieve high spectral efficiencies in future optical communication systems, high order modulations are advocated. In $[4,5]$, the authors addressed the optimization of the mapping of the code bits to the modulation bits of the signal constellation for SC-LDPC codes with SDD and SCGLDPC codes with HDD. It is shown in [5] that an optimized bit allocation improves the performance over the baseline sequential allocation at the expense of a marginal increase in complexity. The improvement is especially significant for tailbiting codes, up to the point where they have a comparable gap to capacity as their terminated counterparts, at a lower FEC overhead.

SC codes with HDD have been considered for optical communications in [1, 2, 7-9]. In [8] an ensemble of SC-GLDPC codes based on $\mathrm{BCH}$ component codes was considered, and 


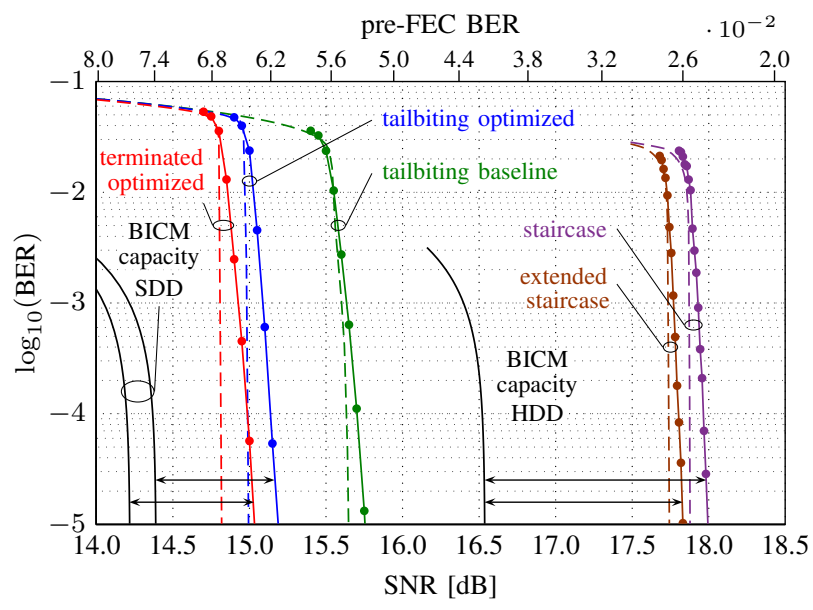

Fig. 3. Simulation (solid curves) and density evolution (dashed curves) results for the SC-LDPC code with 64-QAM over the AWGN channel.

it was shown that iterative HDD can approach capacity in the high-rate regime. In [2] a new extrinsic iterative decoding rule that improves performance for generalized product codes, and a modified construction of TBB codes to reduce decoding delay were proposed. In [9] staircase codes were optimized based on a brute-force search using extensive software simulations. In [7] the authors addressed the optimization of staircase code parameters based on density evolution, a semi-analytical tool that mimics the probabilistic behavior of the decoding process and allows to significantly reduce the optimization time. An extension of staircase codes with larger staircase block sizes, by allowing for multiple code constraints per row/column in the staircase array, was also proposed in [7]. The proposed codes have steeper waterfall performance curves compared to those obtained from the original construction.

In Fig. 3 we give bit error rate (BER) results for SCLDPC codes with SDD and optimized bit mapping [4,5], and for staircase codes [9] and the extended staircase codes proposed in [7], both decoded using HDD, for an additive white Gaussian noise (AWGN) channel. The code rate is 0.75 ( 0.741 for the terminated SC-LDPC code) $(\mathrm{OH}=33 \%)$. The specific code parameters can be found in [4, 5, 7,9]. Staircase codes and extended staircase codes perform 1.5 and $1.3 \mathrm{~dB}$ away from capacity at a BER of $10^{-5}$. An extra coding gain of 2.6-2.8 dB can be achieved by using SC-LDPC codes with optimized bit mapping and SDD (around $0.8 \mathrm{~dB}$ away from capacity), at the expense of a higher complexity and power consumption. However, it is worth pointing out that staircase and extended staircase codes perform closer to capacity for higher rates.

\section{Open Research Problems}

SC codes are very promising candidates to be adopted in future optical communication systems. However, there are still several challenges which need to be addressed before these codes make their way into practical systems. In the following, we provide a partial list of open research problems.

- As discussed in the previous sections, SC codes yield performance very close to the Shannon limit. However, their behavior in the error floor region is not well understood yet. GLDPC codes with HDD show very low error floors, which can be estimated analytically, thanks to their rich algebraic structure. SC-LDPC codes (especially irregular ones), on the other hand, may suffer from a relatively high error floor. The error floor is related to graphical substructures, which depend on parameters such as the lifting factor, the window size, and the adopted finite-precision decoding algorithm. Since optical communications require very low error rates $\left(10^{-15}\right)$, a better understanding of the role of these parameters on the error floor and the impact on the code design is crucial.

- The practical implementation of SC-LDPC codes requires the use of finite-precision decoding algorithms. Furthermore, optical systems have very stringent requirements in terms of power consumption and throughput. No rigorous works on low-complexity decoding algorithms for SCLDPC codes, and their impact on the performance are available yet in the literature. The code design (i.e., the optimization of the code parameters) should consider the use of a low-complexity decoding algorithm.

- Nonlinear effects are arguably one of the most significant challenges that need to be addressed for further increase in capacity of optical core networks. The code designs proposed so far are blind to nonlinearities and have been optimized for either the AWGN or the binary symmetric channel. While simulation results show good performance in the presence of nonlinearities, to fully exploit the potential of FEC in optical communications, a joint design of the FEC code and the modulation scheme, tailored to the nonlinear regime, should be considered.

\section{REFERENCES}

[1] B. P. Smith, A. Farhood, A. Hunt, F. R. Kschischang, and J. Lodge, "Staircase codes: FEC for $100 \mathrm{~Gb} / \mathrm{s}$ OTN," J. Lightwave Technol., vol. 30, no. 1, pp. 110-117, Jan. 2012.

[2] Y.-Y. Jian, H. D. Pfister, K. R. Narayanan, R. Rao, and R. Mazahreh, "Iterative hard-decision decoding of braided $\mathrm{BCH}$ codes for high-speed optical communication," in Proc. IEEE Global Telecom. Conf., Dec. 2013.

[3] S. Kudekar, T. Richardson, and R. Urbanke, "Threshold saturation via spatial coupling: Why convolutional LDPC ensembles perform so well over the BEC," IEEE Trans. Inf. Theory, vol. 57, no. 2, pp. 803-834, Feb. 2011.

[4] C. Häger, A. Graell i Amat, F. Brännström, A. Alvarado, and E. Agrell, "Improving soft FEC performance for higher-order modulations via optimized bit channel mappings," Opt. Express, vol. 22, no. 12, pp. 544 558, Jun. 2014.

[5] - "Terminated and tailbiting spatially-coupled codes with optimized bit mappings for spectrally efficient fiber-optical systems," IEEE/OSA J. Lightwave Technol., 2015.

[6] L. Schmalen, V. Aref, J. Cho, D. Suikat, D. Rösener, and A. Leven, "Spatially coupled soft-decision forward error correction for future lightwave systems," IEEE/OSA J. Lightwave Technol., 2015.

[7] C. Häger, A. Graell i Amat, H. D. Pfister, A. Alvarado, F. Brännström, and E. Agrell, "On parameter optimization for staircase codes," in Proc. OSA Optical Fiber Communication Conference, Mar. 2015.

[8] Y.-Y. Jian, H. D. Pfister, and K. R. Narayanan, "Approaching capacity at high rates with iterative hard-decision decoding," in IEEE Int. Symp. on Information Theory (ISIT), July 2012, pp. 2696-2700.

[9] L. M. Zhang and F. R. Kschischang, "Staircase codes with $6 \%$ to $33 \%$ overhead," IEEE/OSA J. Lightwave Technol., vol. 32, no. 10, pp. 19992002, May 2014 